\title{
Occurrence of Winter Cereal Viruses in New South Wales, Australia, 2006 to 2014
}

Andrew Milgate and Dante Adorada, New South Wales (NSW) Department of Primary Industries, Wagga Wagga Agricultural Institute, Wagga Wagga NSW 2650 Australia; and Grant Chambers and Mary Ann Terras, NSW Department of Primary Industries, Elizabeth Macarthur Agricultural Institute, Menangle, NSW 2568 Australia

\begin{abstract}
Milgate, A., Adorada, D., Chambers, G., and Terras, M. A. 2016. Occurrence of winter cereal viruses in New South Wales, Australia, 2006 to 2014. Plant Dis. 100:313-317.

Winter cereal viruses can cause significant crop losses; however, detailed knowledge of their occurrence in New South Wales, Australia is very limited. This paper reports on the occurrence of Wheat streak mosaic virus (WSMV), Wheat mosaic virus (WMoV), Barley yellow dwarf virus (BYDV), Cereal yellow dwarf virus (CYDV), and their serotypes between 2006 and 2014. Detection of WMoV is confirmed in eastern Australia for the first time. The BYDV and CYDV 2014 epidemic is examined in detail using 139 samples of wheat, barley, and oat surveyed

from southern New South Wales. The presence of virus was determined using enzyme-linked immunosorbent assays. The results reveal a high frequency of the serotype Barley yellow dwarf virus - MAV as a single infection present in $27 \%$ of samples relative to Barley yellow dwarf virus - PAV in 19\% and CYDV in 14\%. Clear differences emerged in the infection of different winter cereal species by serotypes of BYDV and CYDV. These results are contrasted to other Australian and international studies.
\end{abstract}

Records on the incidence of Barley yellow dwarf virus (BYDV), Cereal yellow dwarf virus (CYDV), and their serotypes in winter cereal crops in eastern Australia have been infrequent and limited in their scope. Grass species which host the cereal-infecting viruses and act as reservoirs of disease in Australia have been reviewed recently by Jones (2013). The last published reports of winter cereal viruses in eastern Australia for BYDV and CYDV were in Queensland 1988, New South Wales in 1988, and South Australia in 1992 (Greber 1988; Henry et al. 1992; Sward and Lister 1988). More recently, Wheat streak mosaic virus (WSMV) was identified and confirmed for the first time in eastern Australia in 2002 and Wheat mosaic virus (WMoV), previously known as High plains virus (HPV), was reported in 2006 but unconfirmed in New South Wales and then confirmed in Western Australia in 2013 (Coutts et al. 2008, 2013; Ellis et al. 2003; Murray 2006). However, since these first reports, no large-scale survey for their incidence has been published. When virus-like symptoms developed in winter cereals over a large area of the southern New South Wales cropping zone in 2014, a survey was undertaken to determine the frequency of virus infection and the species and serotypes involved, and compare this with historical records.

BYDV and CYDV are part of the Luteoviridae family of viruses but are from different genera (Luteovirus and Polerovirus, respectively) (Jones 2013; Mayo and D'Arcy 1999). Serotypes known to occur in Australia on perennial grasses and winter cereal crops are Barley yellow dwarf virus - PAV (BYDV-PAV), Barley yellow dwarf virus - MAV (BYDV-MAV) and Cereal yellow dwarf virus - RPV (CYDV-RPV) (Guy et al. 1987; Hawkes and Jones 2005; Henry et al. 1992; McKirdy and Jones 1993; Sward and Lister 1988). WSMV is a member of the Tritimovirus genus in the family Potyviridae and Australian isolates have been identified as belonging to the D1 subclade (Dwyer et al. 2007). WMoV (syn. HPV) is a member of the Emaravirus genus in the Bunyaviridae family (Skare et al. 2006; Tatineni et al. 2014).

Viruses can cause significant crop losses and influence the choice of crop species and variety in areas where they occur regularly. Yield losses are most severe when infection occurs during the early growth

Corresponding author: A. Milgate; E-mail: andrew.milgate@dpi.nsw.gov.au Accepted for publication 3 September 2015.

http://dx.doi.org/10.1094/PDIS-06-15-0650-RE

(C) 2016 The American Phytopathological Society of the seedlings (Banks et al. 1995; McKirdy et al. 2002). The vectors of BYDV and CYDV in Australia are Rhopalosiphum padi (oat aphid) and $R$. maidis (corn aphid); these are the most abundant aphid species colonizing cereals but $R$. insertum, $R$. rufiabdominalis, and Sitobion miscanthi are also found in southwestern Australia (Jones 2013). The vector of WSMV and WMoV is the wheat curl mite (WCM) (Aceria tosichella Keifer), which occurs in Australia in two genetically distinct populations (Schiffer et al. 2009). The lineage which has been demonstrated to transmit WSMV, WCM2, is common in the New South Wales cropping regions (Schiffer et al. 2009).

The 2014 survey focused on southern New South Wales, which is an important winter cereal production area of Australia covering up to 3 million ha, with average crop plantings of 1.4 million ha of wheat, 300,000 of barley, and 175,000 for oat (Scott 2012). The region is characterized by a general trend of increasing rainfall from west to east, the western districts having low annual rainfall of less than $400 \mathrm{~mm}$ and the eastern districts of the cropping region reaching annual rainfall above $600 \mathrm{~mm}$. Sowing occurs in the autumn and is driven by prevailing seasonal conditions of moisture availability and can extend from February to July.

The records of winter cereal virus detection in New South Wales between 2006 and 2013 are presented and discussed. In addition to the historical records, we conducted a survey of wheat, barley, and oat crops in southern New South Wales for the presence of four viruses in the winter and spring of 2014. The number of locations sampled was 139 paddocks and the results of enzyme-linked immunosorbent assay (ELISA) analyses for BYDV (PAV and MAV), CYDV, WSMV, and $\mathrm{WMoV}$ and their spatial distributions are discussed.

\section{Methods and Materials}

Historical virus detection 2006 to 2013. The data presented in Table 1 are from winter cereal samples submitted to the New South Wales Department of Primary Industries (DPI) Plant Health Diagnostic Service from 2006 to 2013. It is not a planned survey and reflects observations made by private owners, agronomists, and New South Wales DPI staff that a winter cereal crop may have been infected with a virus. The following viruses were tested for in each year: BYDV, CYDV, WSMV, and WMoV. However, not all viruses were tested for in all samples. The number of positive test results for each virus or serotype is shown in Table 1, with the total number of tests conducted for each virus or serotype in parentheses. In all cases, BYDV tests from 2006 to 2013 are only for serotype PAV; testing for MAV commenced in 2014.

Survey sample collection 2014. Winter cereal crops of wheat (Triticum aestivum L.), barley (Hordeum vulgare L.), and oat (Avena 
sativa L.) were sampled between May and November 2014. The area sampled extended from Parkes in central New South Wales to Echuca in the south, Griffith to the west, and Goulburn to the east (Fig. 1). A minimum of five plants was sampled within the individual crops from areas displaying virus-like symptoms. For wheat and barley, these symptoms were plants displaying leaves with yellowing from the tips and yellow stripes extending down the leaf blade whereas, for oat, the symptoms targeted were plants displaying red and yellowing leaves. In total, 139 samples were collected and analyzed. The number of samples from wheat, barley, and oat are provided in Table 2.

ELISA. To test for the presence of virus, an ELISA was performed using Agdia commercial kits (Agdia Elkhart) according to the kit protocols, as follows: for WSMV, Agdia catalog number SRA47001; for BYDV using BYDV-PAV, BYDV-MAV, and CYDV-RPV, Agdia catalog numbers SRA27500, SRA26500, and SRA28001, respectively; and, for WMoV (HPV), Agdia catalog numberSRA17200. Serological tests were not conducted for BYDV-RMV because a specific antiserum was not available.

Each sample was tested in duplicate and all test plates contained at least one positive and one negative control and buffer blanks. The negative controls included one cereal species (wheat or barley). Tests were considered positive when the readings for absorbance at $405 \mathrm{~nm}$ were two times greater than the mean of the negative controls. For $\mathrm{WMoV}$, further evidence of its presence was sought by performing reverse-transcription polymerase chain reaction (RT-PCR) on five wheat samples from the 2009 positive results. In addition, one isolate was sequenced using the primers and methods outlined by Lebas et al. (2005) and the sequence data deposited in GenBank. For the other viruses, confirmation was by electron microscopy or immunoelectron microscopy (data not shown).

Mapping. Spatial representation of the collection locations and serotype occurrence (Fig. 1) were constructed using the ggmap package in R (Kahle and Wickham 2013). Sample GPS co-ordinates have been off-set in the figure to allow visualization where points overlapped or where the sample location was mapped to the nearest town, not the precise paddock location.

\section{Results}

The presence of viruses BYDV (PAV and MAV), CYDV (RPV), WSMV, and WMoV were confirmed with positive ELISA tests. In addition to the ELISA tests, the presence of WMoV was confirmed in five wheat samples from the 2009 positive ELISA results by RT-PCR. Further evidence was provided by sequencing one isolate, 09WYPT26. The sequence data were deposited in GenBank, accession

Table 1. Historical occurrence of cereal infecting viruses Barley yellow dwarf virus (BYDV) PAV, Cereal yellow dwarf virus (CYDV) RPV, Wheat streak mosaic virus (WSMV), and Wheat mosaic virus (WMoV) in New South Wales, Australia from 2006 to $2013^{\text {a }}$

\begin{tabular}{|c|c|c|c|c|c|c|c|}
\hline \multirow[b]{2}{*}{ Year, species } & \multirow[b]{2}{*}{ Total tested } & \multicolumn{3}{|c|}{ BYDV/CYDV } & \multirow[b]{2}{*}{ WSMV } & \multirow[b]{2}{*}{ WMoV } & \multirow[b]{2}{*}{ WSMV + WMoV } \\
\hline & & PAV & CYDV & PAV+CYDV & & & \\
\hline \multicolumn{8}{|l|}{2006} \\
\hline Wheat & 101 & - & - & - & $38(101)$ & $1(84)$ & $23(84)$ \\
\hline Barley & 6 & $1(1)$ & - & - & $1(5)$ & $0(5)$ & $0(5)$ \\
\hline Oat & 1 & $0(1)$ & $0(1)$ & $0(1)$ & $0(1)$ & $0(1)$ & $0(1)$ \\
\hline Combined & 108 & 1 & 0 & 0 & 39 & 1 & 23 \\
\hline \multicolumn{8}{|l|}{2007} \\
\hline Wheat & 37 & $0(9)$ & - & - & $2(32)$ & $0(5)$ & 0 \\
\hline Barley & 4 & $1(4)$ & - & - & $1(3)$ & $0(1)$ & 0 \\
\hline Oat & 3 & $0(2)$ & - & - & $0(3)$ & - & - \\
\hline Combined & 44 & 1 & - & - & 3 & 0 & 0 \\
\hline \multicolumn{8}{|l|}{2008} \\
\hline Wheat & 62 & $0(12)$ & $0(4)$ & $0(4)$ & $26(62)$ & $1(43)$ & $4(43)$ \\
\hline Barley & 3 & $1(2)$ & - & - & $1(1)$ & $0(1)$ & $0(1)$ \\
\hline Oat & 2 & $0(2)$ & - & - & $1(2)$ & $0(1)$ & $0(1)$ \\
\hline Combined & 67 & 1 & 0 & 0 & 28 & 1 & 4 \\
\hline \multicolumn{8}{|l|}{2009} \\
\hline Wheat & 151 & $1(59)$ & $0(57)$ & $0(57)$ & 64 (128) & $0(125)$ & $5(125)$ \\
\hline Barley & 6 & $1(12)$ & $0(12)$ & $1(12)$ & $1(2)$ & $0(2)$ & $0(2)$ \\
\hline Oat & 1 & $3(9)$ & $0(9)$ & $0(9)$ & $0(6)$ & $0(3)$ & $0(3)$ \\
\hline Combined & 158 & 5 & 0 & 1 & 65 & 0 & 5 \\
\hline \multicolumn{8}{|l|}{2010} \\
\hline Wheat & 26 & $1(24)$ & $0(20)$ & $2(20)$ & $2(14)$ & $0(8)$ & $0(8)$ \\
\hline Barley & 9 & $2(9)$ & $0(9)$ & $0(9)$ & $0(9)$ & $0(3)$ & $0(3)$ \\
\hline Oat & 13 & $1(13)$ & 7 (13) & $4(13)$ & $0(6)$ & $0(1)$ & $0(1)$ \\
\hline Combined & 48 & 4 & 7 & 6 & 2 & 0 & 0 \\
\hline \multicolumn{8}{|l|}{2011} \\
\hline Wheat & 3 & $1(3)$ & $0(3)$ & $0(3)$ & $1(3)$ & $0(3)$ & $0(3)$ \\
\hline Barley & - & - & - & - & - & - & - \\
\hline Oat & 2 & $0(2)$ & $0(2)$ & $0(2)$ & - & - & - \\
\hline Combined & 5 & 1 & 0 & 0 & 1 & 0 & 0 \\
\hline \multicolumn{8}{|l|}{2012} \\
\hline Wheat & 5 & $1(4)$ & $1(4)$ & $0(4)$ & $0(5)$ & $0(4)$ & $0(4)$ \\
\hline Barley & - & - & - & - & - & - & - \\
\hline Oat & 2 & $2(2)$ & $0(2)$ & $0(2)$ & - & - & - \\
\hline Combined & 7 & 3 & 1 & - & - & - & - \\
\hline \multicolumn{8}{|l|}{2013} \\
\hline Wheat & 14 & $6(14)$ & $1(14)$ & $0(14)$ & $0(14)$ & $0(13)$ & $0(13)$ \\
\hline Barley & 1 & $0(1)$ & $0(1)$ & $0(1)$ & $0(1)$ & $0(1)$ & $0(1)$ \\
\hline Oat & 4 & $2(4)$ & $1(4)$ & $1(4)$ & $0(1)$ & $0(1)$ & $0(1)$ \\
\hline Combined & 19 & 8 & 2 & 1 & - & - & - \\
\hline Total & 456 & 24 & 10 & 8 & 138 & 2 & 32 \\
\hline
\end{tabular}

a Numbers in parentheses indicate the total number of samples tested for each virus. 
number KT013206, which show that it has a $99.67 \%$ homology to accession number U60141-1.

The occurrence of BYDV and CYDV between 2006 and 2012 show that PAV and CYDV have been at low frequency in the samples tested, ranging from as little as a single detection in 2006 to 17 detections (40\%) in 2010 (Table 1). However, in 2013, there is a higher frequency of PAV and CYDV, with $58 \%$ of samples tested returning a positive result. For WSMV and WMoV, the pattern of detection for the same period is characterized by spikes in frequency during 2006, 2008, and 2009. WSMV and WMoV were not detected in 2012 to 2013; however, this is based on limited samples submitted for testing.

There were also no detections of WSMV or WMoV in the 139 samples collected during the 2014 survey, indicating that these viruses were not widespread. However, 53\% of samples were found to be infected with either BYDV or CYDV. The proportion of infected samples within crop species was similar for wheat and barley, with 49 and $50 \%$ infected, respectively. In the oat samples, $100 \%$ infection was detected; however, this was from a smaller sample size $(n=8)$ compared with that of wheat $(n=87)$ and barley $(n=44)$.

Considering the samples which tested positive for BYDV or CYDV in 2014, the most common serotype was MAV (27\%), followed by PAV (19\%), then CYDV (14\%) (Table 2). The serotypes were also detected in combination; the most frequent combination was that of PAV+MAV in $19 \%$ of all samples followed by PAV+CYDV (14\%), while the MAV+CYDV combination was detected in only $3 \%$ of samples. The combination of all three serotypes in the same sample also occurred at low frequency; it was detected in $4 \%$ of the samples.

In the 2014 infected samples, there were differences in the frequency of the BYDV and CYDV serotypes occurring on the different crop species (Table 2). For wheat, the most common serotype was MAV (30\%) and the combination of PAV+MAV (30\%). PAV and CYDV were less common, being detected in $12 \%$ of wheat samples. The combination of MAV+CYDV was the least frequent, found in only $2 \%$ of samples. Barley had a different pattern of serotype infection from that of wheat and oat. The most frequently detected serotype was PAV, in $41 \%$ of barley samples that tested positive. The next most common was the combination of PAV+CYDV, in $27 \%$ of samples. MAV (9\%) and PAV+MAV+CYDV (5\%) were the least common. Two combinations (PAV+MAV and MAV+CYDV) were not detected in the barley samples.

The number of oat samples tested was less than the other two crop species; however, $100 \%$ of the samples tested returned positive results. The pattern of serotype infection in these samples contrasted with that in wheat and barley. The most common serotype was MAV, in $64 \%$ of the samples; the next most frequent, in $12 \%$ of samples each, were CYDV, PAV+MAV, and MAV+CYDV. PAV was not detected in any of the samples either alone or in combination with MAV or CYDV.

The distribution of sample locations for wheat and oat crops were spread evenly across southern New South Wales (Fig. 1). Barley crop sampling did not extend south beyond Mangoplah. Positive results were found to occur across the entire region. The distributions of the serotypes found infecting wheat show some spatial patterns (Fig. 1). MAV appeared dominant in the Parkes and Forbes area, becoming less frequent as a single serotype infection in the Wagga Wagga area. In the Wagga Wagga area, MAV was only detected in combinations with PAV and PAV+CYDV. For PAV and CYDV, the pattern was the opposite of MAV, being dominant in the Wagga Wagga area either on its own or in combination with MAV. No positive wheat samples were detected south of Mangoplah in southern New South Wales.

For barley, the distributions of serotypes appeared more uniform. The individual infections of PAV, MAV, and CYDV or the twoserotype combinations detected occurred evenly across the area of positive samples found (Fig. 1). The lower number of oat crops sampled means that spatial patterns are not distinct. MAV was the most common serotype and was found in all parts of the southern New South Wales region, sampled either on its own or in combination with PAV or CYDV.

\section{Discussion}

This study reports the first comprehensive study of WSMV, WMoV, BYDV, and CYDV and their serotype occurrence in New South Wales. We report here the first confirmed detection of WMoV in eastern Australia. The presence of $\mathrm{WMoV}$ was confirmed in Western Australia in 2013; however, in eastern Australia, WMoV was reported as being present in a New South Wales government department report but has, until now, not been confirmed in the published literature (Coutts et al. 2014; Ellis et al. 2003; Murray 2006). The results in Table 1 show that $\mathrm{WMoV}$ has been present in New South Wales since at least 2006, when testing began for this virus. WMoV was rarely detected as a sole infection and was most frequently associated with WSMV infections. This association with WSMV has been documented in other studies (Coutts et al. 2014; Navia et al. 2013; Skare et al. 2006). It is likely that WMoV has been present in Australia as long as WSMV; however, its presence went undetected because its symptoms and occurrence are cryptic with those of WSMV infections.

WSMV and WMoV have not been detected in New South Wales since 2011. Neither were they detected during the 2014 survey of crops with virus-like symptoms discussed here in detail. The causes for the current low incidence of these viruses is not known. The vector is known to be common in the New South Wales grain-growing regions (Schiffer et al. 2009). The environmental conditions required for the build-up and survival of the WCM populations were met during the 2014 and 2013 seasons. Dramatic changes have not occurred in the resistance levels of the wheat, barley, and oat varieties grown in New South Wales which could explain a decline in incidence. Similarly changes have not occurred in the farming system practices or the abundance of alternate weedy hosts over this time period. Thus, there are other undetermined factors which have led to the apparent low incidence of WSMV and WMoV at this point in New South Wales.

This study provides new evidence on the occurrence of BYDV and CYDV in the winter cereals wheat, barley, and oat in Australia. Many studies in Australia on the occurrence of BYDV and CYDV have focused on the alternate host grass species rather than crops species (Hawkes and Jones 2005; Jones 2013; McKirdy and Jones 1993; Sward and Lister 1988). The only report which includes samples from New South Wales, Sward and Lister (1988), tested eight sites and failed to detect BYDV in wheat or barley and found only one positive sample of oat, while surveys by Greber (1988) of wheat in southeastern Queensland found $16 \%$ of wheat and barley samples infected and, in Victoria, Sward and Lister (1987) found 38\% of wheat samples infected with BYDV.

For BYDV and CYDV, there are a number of differences between our findings and those of the previous surveys of viruses in eastern Australia. Our 2014 survey has found that MAV was the most frequent serotype overall, followed by PAV, during the 2014 epidemic. PAV was found to be the most frequent serotype, followed by CYDV in Victoria from 15 sites (Sward and Lister

Table 2. Frequency of Barley yellow dwarf virus serotype (MAV and PAV) or Cereal yellow dwarf virus serotype (RPV) infections on winter cereal species wheat, barley, and oat in southern New South Wales during 2014

\begin{tabular}{lcccc}
\hline & \multicolumn{3}{c}{ Crop species (\%) } & \\
\cline { 2 - 4 } Serotype & Barley & Oat & Wheat & Combined (\%) \\
\hline PAV & 41 & 0 & 12 & 19 \\
MAV & 9 & 64 & 30 & 27 \\
CYDV & 18 & 12 & 12 & 14 \\
PAV+MAV & 0 & 12 & 30 & 19 \\
PAV+CYDV & 27 & 0 & 9 & 14 \\
MAV+CYDV & 0 & 12 & 2 & 3 \\
PAV+MAV+CYDV & 5 & 0 & 5 & 4 \\
Positive/total & $22 / 44$ & $8 / 8$ & $43 / 87$ & $73 / 139$ \\
\hline
\end{tabular}

a Virus detection was carried out using enzyme-linked immunosorbent assay.

b Number of samples positive/total tested. 
1987) . In South Australia, PAV was the most common serotype in wheat; however, the authors did not test for the presence of MAV (Henry et al. 1992). Similarly, PAV was also the most common serotype found in a national survey comprising 31 sites conducted in 1988 (Sward and Lister 1988). However, these samples contained only one positive result from New South Wales in an oat crop. This was insufficient sampling to draw conclusions on the incidence of serotypes in New South Wales.

Distinct patterns of serotype incidence emerged between the different species of crops surveyed. PAV was the most abundant found in barley crops, followed by CYDV. This pattern is similar to the observations reported by Sward and Lister (1987) and Sward and Lister (1988) on winter cereal crops. Also, PAV has been recorded as being the most common serotype on pasture and grass species in Australia (Greber 1988; Guy et al. 1987). In contrast, for wheat, MAV was the most common serotype, at $30 \%$ of infected samples in this current study. This suggests that MAV has either become more common in eastern Australia since the 1980s or that we have been able to reveal differences in feeding preferences of the aphid vectors not previously detected. These apparent differences from older studies could be explained by the higher number of naturally infected crops detected in this current study.

The high frequency of MAV is also different when compared with other international studies on the occurrence of BYDV serotypes. In Latvian barley and oat crops, PAV was the most abundant, followed by MAV, and combinations were less common (Bisnieks et al. 2006). In Iranian fields of wheat and barley crops, PAV was the most abundant, followed by CYDV, then MAV (Pakdel et al. 2010). In contrast, in Hungary, the frequency of infections in wheat and barley crops were PAV as the most abundant, followed by RMV, then CYDV, and no MAV was detected (Pocsai et al. 1995).

Further differences from previous surveys are found in the high frequency of serotype mixtures detected in this current study. Mixtures of serotypes were found in $40 \%$ of the samples tested in the current study; this is 31\% higher than that recorded in Victoria by Sward and Lister (1988), who reported only a 9\% occurrence of mixtures. In the current study, the most common mixture was PAV+MAV in 19\% of samples, followed by PAV+CYDV in $14 \%$ (Table 2). Sward and Lister (1988) found that the PAV+CYDV combination was the most common but also noted that PAV+MAV was common as well. The frequency of mixtures supports the conclusion of Guy et al. (1987) that these serotypes are cotransmitted by aphid vectors from grass hosts also infected with mixtures.

The extent of BYDV and CYDV infection across the region observed in 2014 is considered higher than that normally expected in southern New South Wales. The records of viruses in 2006 to 2013 (Table 1) indicate that a build-up of BYDV and CYDV had most likely commenced during 2013, providing the reservoir from which the 2014 epidemic could begin. This reservoir of virus combined with two other factors resulted in the epidemic. First, the

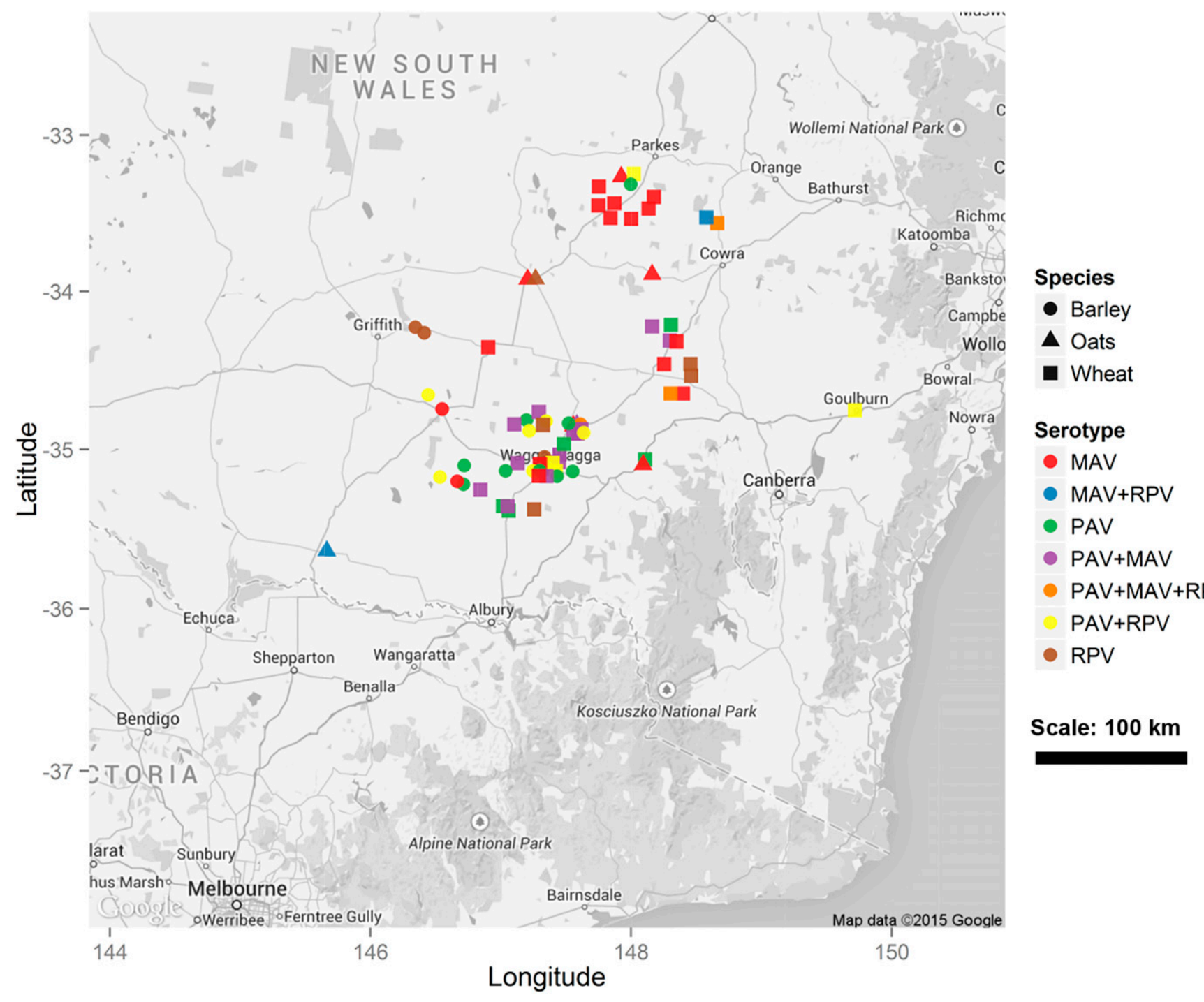

Fig. 1. Map of locations winter cereal crops sampled in New South Wales, Australia during 2014 and tested positive for the presence of Barley yellow dwarf virus (MAV and PAV) and Cereal yellow dwarf virus (RPV). 
prevailing mild weather conditions extending from autumn to winter (March to June), allowing aphid numbers to increase rapidly and move over a wide area unchecked by temperature stress. Second, early seasonal rainfall events in late summer and early autumn (February and March) promoted pasture growth after a dry summer period as well as early sowing of susceptible winter cereals, providing ample resources for aphids to feed and multiply. The rapid spread of viruses in 2014 show the dynamic nature of the vector populations and their threat to cropping.

Mapping of the occurrence of serotypes reveals clear differences in the spatial patterns of infections and, thus, the feeding preferences of aphids between barley and wheat. In the Wagga Wagga area, there were frequent infections of wheat with the combination of PAV+ MAV. However, barley crops in the same area had no infections of PAV+MAV detected. Although the aphid vector species on samples were not recorded in this study, the infection patterns can be compared with the published records and transmission studies. The vectors most likely to be responsible for barley infections were the oat aphid. This is supported by Jones (2013), who states that the oat aphid regularly transmits PAV and CYDV but rarely MAV. For wheat, several vectors may have been involved: the grain aphid or rose-grain aphid as the main vectors but also the oat aphid (Jones 2013). The grain and rose-grain aphids both have been shown to regularly transmit PAV and MAV and, rarely, CYDV (Jones 2013; Sward and Lister 1988).

Limited oat samples were processed; however, no PAV on its own or in combinations with CYDV was detected, which is not consistent with published reports of transmission from Jones (2013) or Sward and Lister (1988). PAV was common in barley and, to a lesser extent, in wheat, indicting that transmission from these PAV-infected crops to oat was not frequent. A possible explanation for the observed pattern of infections between the crop species is localized feeding preferences of the aphid vectors. Recently, a hypothesis of vector manipulation by the virus has been proposed by Ingwell et al. (2012) to explain the spread of viruses such as BYDV. They demonstrated that, once aphids acquire the virus, they will preferentially feed on noninfected plants, which would significantly affect the spread of virus to noninfected host plants within a crop and surrounding crops (Ingwell et al. 2012). This hypothesis could be extended to help explain the spatial pattern and apparent preferential feeding of virus-infected aphids observed as the distinct distribution of serotypes in the current study.

In conclusion, we have presented new evidence of the occurrence of winter cereal viruses in eastern Australia. This is the first confirmed report of WMoV in eastern Australia and the first comprehensive survey of BYDV and CYDV viruses and their serotypes in New South Wales showing historical trends. These results have highlighted that BYDV and CYDV are significant diseases in the grain-growing regions and can rapidly expand their area of impact under favorable conditions. The presence of different serotypes and their implications for management and resistance breeding requires further research. This study provides the first step by identifying which serotypes of the viruses are present and their relative abundance.

\section{Acknowledgments}

This study was funded by New South Wales Department of Primary Industries and Grains Research and Development Corporation project DAN00177. We thank the many agronomists and growers who assisted with the survey and submitted historical samples; and W. Forbes, S. Austin, B. Gorrie, A. Ghalayini, and J. Gray for their technical assistance in processing the samples or collating the data.

\section{Literature Cited}

Banks, P. M., Davidson, J. L., Bariana, H., and Larkin, P. J. 1995. Effects of barley yellow dwarf virus on the yield of winter-wheat. Aust. J. Agric. Res. 46:935-946.
Bisnieks, M., Kvarnheden, A., Turka, I., and Sigvald, R. 2006. Occurrence of Barley yellow dwarf virus and Cereal yellow dwarf virus in pasture grasses and spring cereals in Latvia. Acta Agric. Scand. Sect. B Soil Plant Sci. 56: 171-178.

Coutts, B. A., Cox, B. A., Thomas, G. J., and Jones, R. A. C. 2013. First report of Wheat mosaic virus infecting wheat in Western Australia. Plant Dis. 98:285.

Coutts, B. A., Hammond, N. E. B., Kehoe, M. A., and Jones, R. A. C. 2008 Finding Wheat streak mosaic virus in south-west Australia. Aust. J. Agric. Res. 59:836-843.

Dwyer, G. I., Gibbs, M. J., Gibbs, A. J., and Jones, R. A. C. 2007. Wheat streak mosaic virus in Australia: Relationship to isolates from the Pacific Northwest of the USA and its dispersion via seed transmission. Plant Dis. 91:164-170.

Ellis, M. H., Rebetzke, G. J., Mago, R., and Chu, P. 2003. First report of Wheat streak mosaic virus in Australia. Australas. Plant Pathol. 32:551-553.

Greber, R. 1988. Ecology of barley yellow dwarf viruses in south-east Queensland. Australas. Plant Pathol. 17:101-104.

Guy, P., Johnstone, G., and Morris, D. 1987. Barley yellow dwarf viruses in, and aphids on, grasses (including cereals) in Tasmania. Aust. J. Agric. Res. 38: 139-152.

Hawkes, J. R., and Jones, R. A. C. 2005. Incidence and distribution of Barley yellow dwarf virus and Cereal yellow dwarf virus in over-summering grasses in a Mediterranean-type environment. Aust. J. Agric. Res. 56: 257-270.

Henry, M., Francki, R. I. B., and Wallwork, H. 1992. Occurrence of barley yellow dwarf virus in cereals and grasses of the low-rainfall wheatbelt of South Australia. Plant Pathol. 41:713-721.

Ingwell, L. L., Eigenbrode, S. D., and Bosque-Perez, N. A. 2012. Plant viruses alter insect behavior to enhance their spread. Sci. Rep. 2:578.

Jones, R. A. C. 2013. Virus diseases of pasture grasses in Australia: Incidences, losses, epidemiology, and management. Crop Pasture Sci. 64:216-233.

Kahle, D., and Wickham, H. 2013. ggmap: Spatial visualization with ggplot2. R J. 5:144-161.

Lebas, B. S. M., Ochoa-Corona, F. M., Elliott, D. R., Tang, Z., and Alexander, B. J. R. 2005. Development of an RT-PCR for High Plains virus indexing scheme in New Zealand post-entry quarantine. Plant Dis. 89:1103-1108.

Mayo, M. A., and D'Arcy, C. J. 1999. Family Luteoviridae: A reclassification of luteoviruses. Pages 15-22 in: The Luteoviridae. H. G. Smith and H. Barker, eds. CABI Publishing, Wallingford, UK.

McKirdy, S. J., and Jones, R. A. C. 1993. Occurrence of barley yellow dwarf virus serotypes MAV and RMV in over-summering grasses. Aust. J. Agric. Res. 44 1195-1209.

McKirdy, S. J., Jones, R. A. C., and Nutter, F. W. 2002. Quantification of yield losses caused by Barley yellow dwarf virus in wheat and oats. Plant Dis. 86: 769-773.

Murray, G. M. 2006. Update on Wheat streak mosaic virus. Plant Disease Notes. New South Wales Department of Primary Industries, Orange, NSW, Australia.

Navia, D., De Mendonca, R. S., Skoracka, A., Szydło, W., Knihinicki, D., Hein, G. L., da Silva Pereira, P. R. V., Truol, G., and Lau, D. 2013. Wheat curl mite, Aceria tosichella, and transmitted viruses: An expanding pest complex affecting cereal crops. Exp. Appl. Acarol. 59:95-143.

Pakdel, A., Afsharifar, A., Niazi, A., Almasi, R., and Izadpanah, K. 2010. Distribution of cereal luteoviruses and molecular diversity of BYDV-PAV isolates in Central and Southern Iran: Proposal of a new species in the genus Luteovirus. J. Phytopathol. 158:357-364.

Pocsai, E., Kovacs, G., Muranyi, I., Orosz, A., Papp, M., and Szunics, L. 1995 Differentiation of barley yellow dwarf luteovirus serotypes infecting cereals and maize in Hungary. Agronomie 15:401-408.

Schiffer, M., Umina, P., Carew, M., Hoffmann, A., Rodoni, B., and Miller, A. 2009. The distribution of wheat curl mite (Aceria tosichella) lineages in Australia and their potential to transmit Wheat streak mosaic virus. Ann. Appl. Biol. 155:371-379.

Scott, F. 2012. NSW Grains Report Summary 1993-2012. NSW Department of Primary Industries, NSW Grains Report, Orange, NSW, Australia.

Skare, J. M., Wijkamp, I., Denham, I., Rezende, J. A. M., Kitajima, E. W., Park, J.-W., Desvoyes, B., Rush, C. M., Michels, G., Scholthof, K.-B. G., and Scholthof, H. B. 2006. A new eriophyid mite-borne membrane-enveloped virus-like complex isolated from plants. Virology 347:343-353.

Sward, R., and Lister, R. 1987. The incidence of barley yellow dwarf viruses in wheat in Victoria. Aust. J. Agric. Res. 38:821-828.

Sward, R., and Lister, R. 1988. The identity of barley yellow dwarf virus isolates in cereals and grasses from mainland Australia. Aust. J. Agric. Res. 39: 375-384.

Tatineni, S., McMechan, A. J., Wosula, E. N., Wegulo, S. N., Graybosch, R. A., French, R., and Hein, G. L. 2014. An eriophyid mite-transmitted plant virus contains eight genomic RNA segments with unusual heterogeneity in the nucleocapsid protein. J. Virol. 88:11834-11845. 